Article

\title{
Dose-Dependent Effects of Dietary Xylooligosaccharides Supplementation on Microbiota, Fermentation and Metabolism in Healthy Adult Cats
}

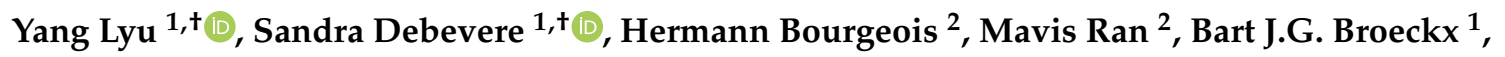 \\ Lynn Vanhaecke ${ }^{3}$, Tom Van de Wiele ${ }^{4}$ and Myriam Hesta ${ }^{2, *}$ \\ 1 Department of Nutrition, Genetics and Ethology, Faculty of Veterinary Medicine, Ghent University, \\ Heidestraat 19, 9820 Merelbeke, Belgium; yang.lyu@ugent.be (Y.L.); sandra.debevere@ugent.be (S.D.); \\ bart.broeckx@ugent.be (B.J.G.B.) \\ 2 Longlive Biotechnology Co. Ltd., Haier Road 63, Qingdao 266103, China; hfmbourgeois@gmail.com (H.B.); \\ mavis_ran@hotmail.com (M.R.) \\ 3 Lab of Chemical Analysis, Faculty of Veterinary Medicine, Ghent University, Salisburylaan 133, \\ 9820 Merelbeke, Belgium; lynn.vanhaecke@ugent.be \\ 4 Center for Microbial Ecology and Technology (CMET), Faculty of Bioscience Engineering, Ghent University, \\ Coupure Links 653, 9000 Ghent, Belgium; tom.vandewiele@ugent.be \\ * Correspondence: myriam.hesta@ugent.be; Tel.: +32(0)92647827 \\ + These authors contributed equally to this work.
}

Academic Editor: Solomon Habtemariam

Received: 6 October 2020; Accepted: 27 October 2020; Published: 29 October 2020

\begin{abstract}
In order to investigate the effect and appropriate dose of prebiotics, this study evaluated the effect of two levels of xylooligosaccharides (XOS) in cats. Twenty-four healthy adult cats were divided into three groups: no-XOS control diet with 1\% cellulose; low XOS supplementation (LXOS) with $0.04 \%$ XOS and $0.96 \%$ cellulose; and high XOS supplementation (HXOS) with $0.40 \%$ XOS and $0.60 \%$ cellulose. Both XOS groups increased blood 3-hydroxybutyryl carnitine levels and decreased hexadecanedioyl carnitine levels. Both XOS treatments displayed an increased bacterial abundance of Blautia, Clostridium XI, and Collinsella and a decreased abundance of Megasphaera and Bifidobacterium. LXOS groups increased fecal $\mathrm{pH}$ and bacterial abundance of Streptococcus and Lactobacillus, decreased blood glutaryl carnitine concentration, and Catenibacterium abundance. HXOS group showed a more distinct microbiome profile and higher species richness, and an increased bacterial abundance of Subdoligranulum, Ruminococcaceae genus (unassigned genus), Erysipelotrichaceae genus, and Lachnospiraceae. Correlations between bacterial abundances and blood and fecal parameters were also observed. In conclusion, XOS could benefit feline gut health by altering microbiota; its effects dependant on the dose. The higher-dose XOS increased bacterial populations that possibly promoted intestinal fermentation, while the lower dose altered populations of carbohydrate-metabolic microbiota and possibly modulated host metabolism. Low-dose prebiotics may become a trend in future studies.
\end{abstract}

Keywords: xylooligosaccharides (XOS); microbiome; gut health; prebiotics; cats

\section{Introduction}

Fermentation of prebiotics results in the production of short-chain fatty acids (SCFAs; acetate, propionate, and butyrate), which are generally believed to be beneficial for the host [1]. Human studies have demonstrated that prebiotics can potentially prevent a wide variety of conditions such as obesity, allergy, and inflammatory diseases, amongst others [2-4]. Many studies have already investigated different types of prebiotics and have demonstrated a variety of beneficial effects of those, including the improvement of insulin sensitivity in dogs and horses (short-chain fructooligosaccharides) $[5,6]$; 
the amelioration of the immune response in piglets (mannan oligosaccharide) [7]; and the better performance in poultry (lactulose) [8].

Xylooligosaccharides (XOS) are polymers of the sugar xylose; they are produced from the xylan fraction in plant fiber [9]. As a class of prebiotics, XOS has been demonstrated to exhibit a great number of health benefits for humans and rodents [10], such as the improvement of bowel function and mineral absorption, the regulation of intestinal microbiota, the reduction in colon cancer risk as well as the enhancement of antioxidant capacity, anti-inflammatory and antimicrobial functions [11,12]. Furthermore, XOS has also been reported to be effective in regulating lipid metabolism, for e.g., reducing the cholesterol and triglycerides in diabetic rats $[13,14]$, whereas Sheu et al. noted an improvement in blood glucose and serum lipids in patients with type 2 diabetes mellitus [15]. Studies in adult humans show that the effective daily dose of XOS is only $1.4 \mathrm{~g} /$ day as compared to fructooligosaccharides and galactooligosaccharides, which have a minimum effective dose of $10.0 \mathrm{~g} /$ day [16-18]. This makes it possible to use lower doses of prebiotics so that side effects (e.g., excessive flatus and borborygmi) and production costs could be reduced. Moreover, its prominent stability to acid and heat compared to other indigestible polysaccharides makes it a promising supplement for pet food [19].

Despite a short colon and the lack of a functional caecum as evolutionary adaptations to a strictly carnivorous diet, considerable microbial fermentation occurs in the hindgut of domestic cats [20]. Microbiota of domestic cats have been demonstrated to be capable of fermenting a broad range of dietary fibers, including prebiotics [21]. The majority of the information on the benefit of fibers or prebiotics is, however, extrapolated from human nutrition. Dietary and metabolic interspecies differences should be considered, as domestic cats, which are obligate carnivores, rely on high protein (HP)-containing animal tissues to meet their unique nutritional requirements and are metabolically adapted to lower glucose utilization and higher protein metabolism [22,23]. In order to explore the effect and appropriate dosage of XOS, this study was established to evaluate the effects of two XOS supplementation levels in cats. The antioxidant status, fermentation pattern, metabolic profile, and microbial composition were sequentially determined in 24 healthy spayed adult cats.

\section{Results}

During the trial, body weight and body condition did not differ among the three groups. All diets were well tolerated and did not markedly affect food intake.

\subsection{Fecal Parameters}

The effect of dietary XOS supplementation on fecal parameters (fecal score, $\mathrm{pH}$ and fermentation pattern) is summarised in Figure 1. Compared with the control group, the low XOS supplementation (LXOS) group had significantly increased fecal $\mathrm{pH}$. There was no significant effect on fecal parameters between two periods, and no diet $\mathrm{x}$ period interaction was observed. Data without significant differences or trends are summarised in Table S1.
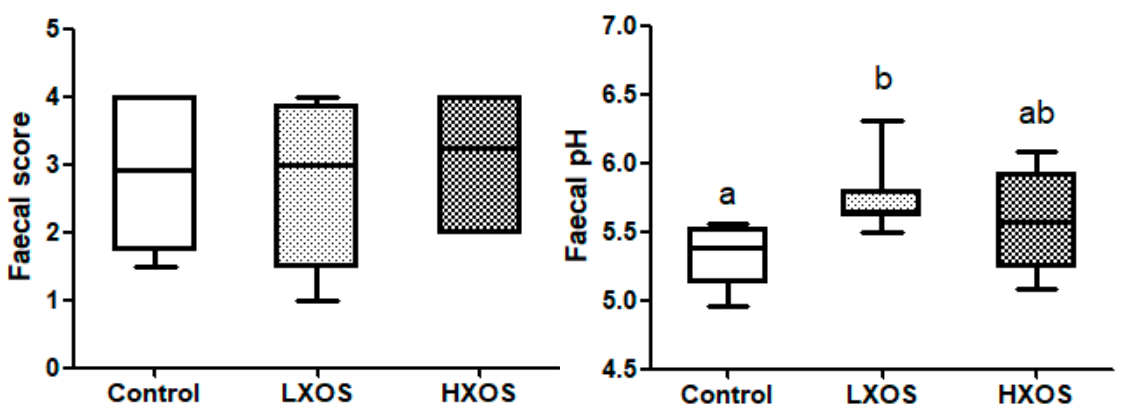

Figure 1. Effect of xylooligosaccharides (XOS) on fecal score and $\mathrm{pH}$. Groups with different letters $(\mathrm{a}, \mathrm{b})$ are significantly different $(p<0.05)$. 


\subsection{Blood Parameters}

The effect of XOS supplementation on blood concentration of 3OH-C4, C5DC, and C16DC is shown in Figure 2. Compared with the control group, both XOS groups significantly increased $3 \mathrm{OH}-\mathrm{C} 4$ concentration and decreased C16DC concentration; the LXOS group had decreased C5DC concentration in blood. No period effect and diet x period interaction was observed on blood parameters. There was no statistical difference or trends in amino acids profiles (Table S1); data without significant differences or trends in acylcarnitine profiles are summarised in Table S1.
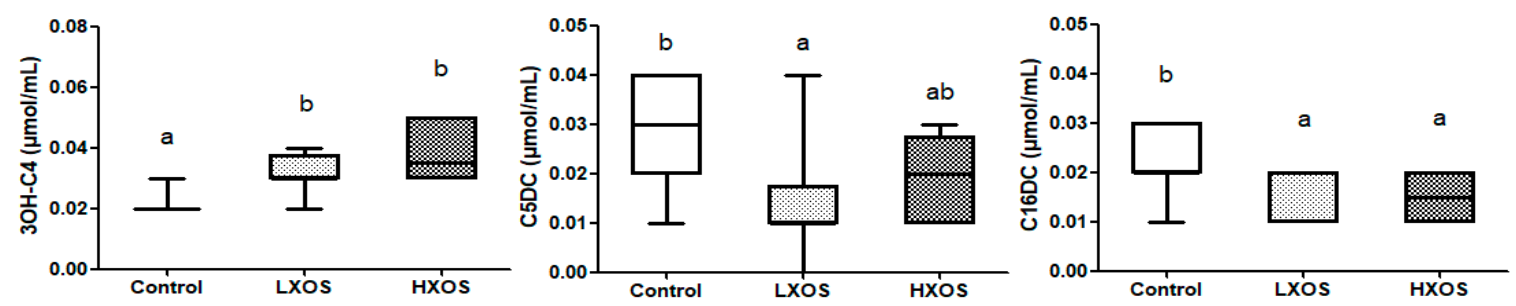

Figure 2. Effect of XOS on the blood concentration of 3OH-C4, C5DC, and C16DC. 3OH-C4: 3-hydroxybutyryl carnitine; C5DC: glutaryl carnitine; C16DC: hexadecanedioyl carnitine. Groups with different letters $(a, b)$ are significantly different $(p<0.05)$.

\subsection{Microbiome Profile}

A total of 367,082 reads were obtained from the fecal samples in the three groups, with 96,089 reads from the control group, 128,427 from the LXOS group, and 142,566 from the high XOS supplementation (HXOS) group. The species richness in HXOS group was significantly higher than that in the control group; there were also trends in the HXOS group of an increased Shannon index and inverse Simpson index (Table 1).

Table 1. Impact of XOS supplementation on diversity, richness, and evenness of the fecal microbiome.

\begin{tabular}{cccccc}
\hline Item. & Control & LXOS & HXOS & SEM & $p$ Value \\
\hline Shannon index & 1.94 & 1.91 & 2.23 & 0.11 & 0.077 \\
Simpson index & 0.78 & 0.758 & 0.838 & 0.03 & 0.185 \\
Inverse Simpson index & 4.86 & 5.05 & 6.49 & 0.583 & 0.061 \\
Pielou's evenness & 0.499 & 0.463 & 0.536 & 0.023 & 0.271 \\
Species richness & $49.4^{\mathrm{a}}$ & $61.6^{\mathrm{ab}}$ & $64.8^{\mathrm{b}}$ & 4.41 & 0.022 \\
\hline
\end{tabular}

$\mathrm{a}, \mathrm{b}$ values within a row with different letters differ $(p<0.05)$. LXOS: low XOS supplementation group; HXOS: high XOS supplementation group.

A heat map with a dimensional cluster dendrogram is presented in Figure 3. From the cluster dendrogram, it is clear that XOS groups did not cluster, indicating a strong animal variability that bigger than the possible differences induced by the dietary treatment. Nevertheless, cats fed with $0.4 \%$ XOS seem to have a more distinct microbiome profile than cats fed with $0.04 \%$ XOS or the control diet. Cats in the control and LXOS groups seemed to have a similar fecal microbiome profile, as they clustered more together. 


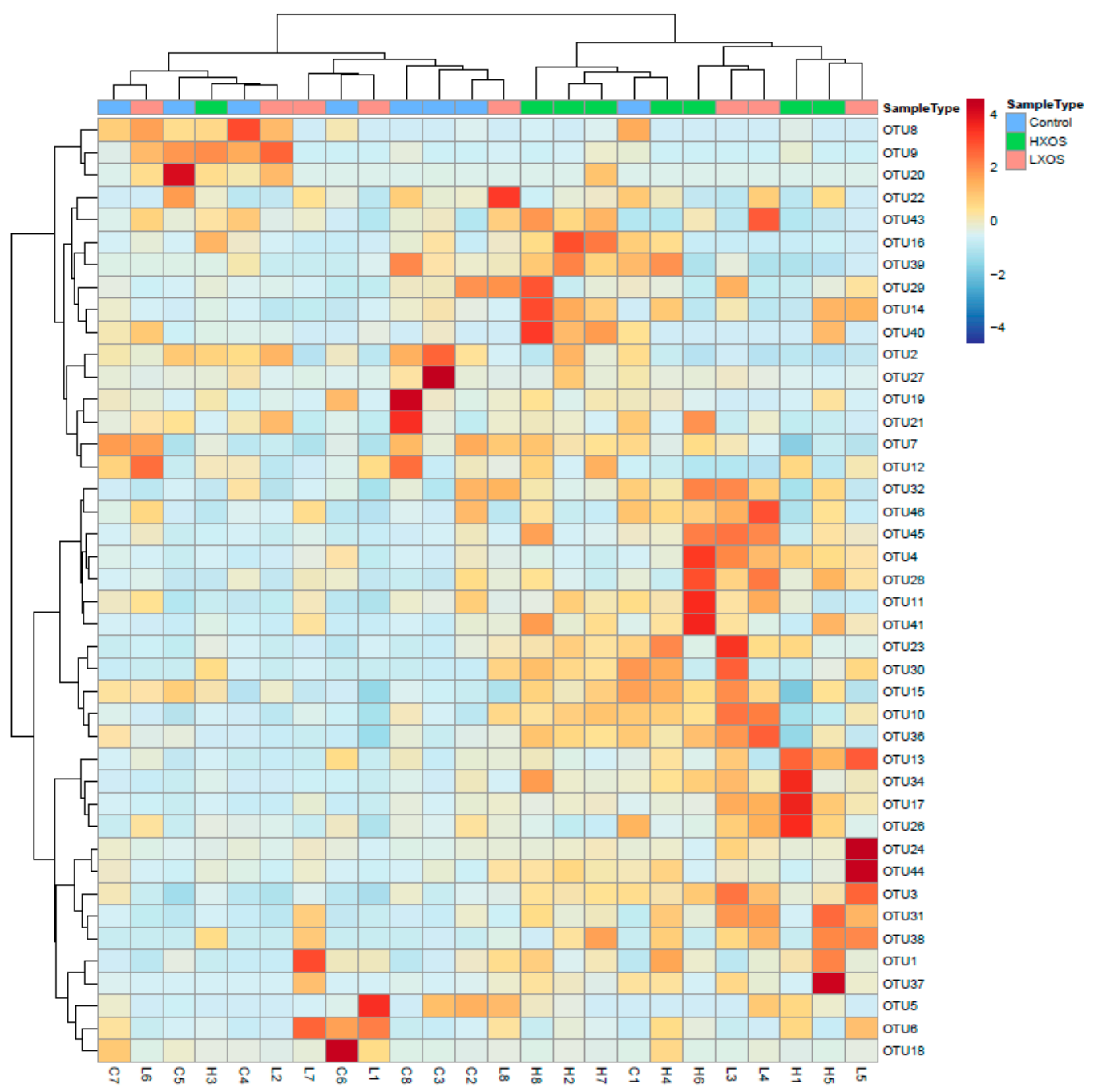

Figure 3. Heat map of the relative abundance and dendrogram clustering of the fecal microbiota genera. Colors with values represent log-transformed relative abundance of operational taxonomic units (OTUs).

The relative abundance of the OTUs (operational taxonomic units) is summarized in Table 2. There were 16 OTUs with a significant difference in the fecal microbiome. Briefly, compared with the control group, both of XOS groups significantly increased the abundance of the genera Blautia, Clostridium XI, and Collinsella and decreased the abundance of Megasphaera and Bifidobacterium $(p<0.05)$; LXOS group increased the abundance of Streptococcus, Lactobacillus, and decreased the abundance of Catenibacterium ( $p<0.05$ ) while HXOS group increased the abundance of Ruminococcaceae genus (an unassigned genus that belongs to the family Ruminococcaceae), Erysipelotrichaceae genus, Subdoligranulum, and Lachnospiraceae genus $(p<0.05)$. 
Table 2. Relative abundance of the operational taxonomic units (OTUs).

\begin{tabular}{|c|c|c|c|c|}
\hline Taxonomy & Control & LXOS & HXOS & $p$ Value \\
\hline \multicolumn{5}{|l|}{ Firmicutes } \\
\hline Bacilli; Lactobacillales; Streptococcaceae; Streptococcus & $13.468^{\mathrm{a}}$ & $28.510^{b}$ & $7.561^{\mathrm{a}}$ & $<0.001$ \\
\hline Clostridia; Clostridiales; Ruminococcaceae; unclassified genus & $1.523^{\mathrm{a}}$ & $0.871^{\mathrm{a}}$ & $4.522^{b}$ & $<0.001$ \\
\hline Clostridia; Clostridiales; Lachnospiraceae; Blautia & $0.396^{\mathrm{a}}$ & $1.077^{\mathrm{b}}$ & $1.870^{\mathrm{c}}$ & $<0.001$ \\
\hline Erysipelotrichia; Erysipelotrichales; Erysipelotrichaceae; unclassified genus & $1.495^{\mathrm{a}}$ & $2.193^{\mathrm{a}}$ & $4.294^{b}$ & 0.001 \\
\hline Clostridia; Clostridiales; Lachnospiraceae; Blautia & $2.024^{\mathrm{a}}$ & $4.051^{\mathrm{b}}$ & $2.085^{\mathrm{a}}$ & 0.029 \\
\hline Clostridia; Clostridiales; Lachnospiraceae; Blautia & $6.423^{\mathrm{a}}$ & $12.713^{\mathrm{b}}$ & $9.363 \mathrm{ab}$ & 0.038 \\
\hline Clostridia; Clostridiales; Lachnospiraceae; unclassified genus & $0.127^{\mathrm{a}}$ & $0.399 \mathrm{ab}$ & $0.505^{b}$ & 0.044 \\
\hline Negativicutes; Selenomonadales; Veillonellaceae; Megasphaera & $1.337^{\mathrm{b}}$ & $0.790^{\mathrm{a}}$ & $0.819^{\mathrm{a}}$ & 0.045 \\
\hline Clostridia; Clostridiales; Peptostreptococcaceae; Clostridium XI & $2.198^{\mathrm{a}}$ & $3.258^{\mathrm{b}}$ & $3.094^{b}$ & 0.047 \\
\hline Actinobacteria; Bifidobacteriales; Bifidobacteriaceae; Bifidobacterium & $14.403^{\mathrm{b}}$ & $16.259^{b}$ & $4.466^{\mathrm{a}}$ & 0.006 \\
\hline Actinobacteria; Bifidobacteriales; Bifidobacteriaceae; Bifidobacterium & $19.854^{b}$ & $5.829^{\mathrm{a}}$ & $7.224^{\mathrm{a}}$ & 0.011 \\
\hline
\end{tabular}

$\mathrm{a}, \mathrm{b}$ values within a row with different letters differ $(p<0.05)$.

To reveal the correlations between microbiota, intestinal fermentation and body metabolism, a Spearman's correlation analysis was performed, and the result is presented in Figure 4. Briefly, Bifidobacterium (OTU 2 and 27) were positively correlated with butyric acid, isobutyrate, isovalerate, and total branched-chain fatty acids (BCFA), negatively correlated with indol and C5:1; Lactobacillus (OTU 1, 9 and 18) were positively correlated with butyric acid, indol, C2, C5:1, and Met, negatively correlated with C4, C5:1, Fen, and Ala; Catenibacterium (OTU19) was positively correlated with Cit and negatively correlated with C5:1; Blautia (OTU 3 and 12) were positively correlated with indol, butyric acid, total SCFA, C5:1, and 3OH-C4; Ruminococcaceae genus (OTU 16 and 30) were positively correlated with butyric acid, isobutyrate, isovalerate, ammonia, total BCFA Orn, Cit and 3OH-C4, negatively correlated with phenol; Collinsella (OTU 7 and 10) were positively correlated with indol, acetic acid, isobutyrate, isovalerate, total BCFA, Orn, Cit C0, C5:1 and 3OH-C4; Subdoligranulum (OTU23) was positively correlated with indol, isobutyrate, isovalerate, total BCFA, $\mathrm{C} 5: 1$ and 3OH-C4; Lachnospiraceae genus (OTU 15 and 24) were positively correlated with indol, propionic acid, Orn, Fen, C0, C5:1 and 3OH-C4; Erysipelotrichaceae genus (OTU14) were positively correlated with Orn, Cit and 3OH-C4; Streptococcus (OTU5) were positively correlated with C5DC; Clostridium XI (OTU4) were positively correlated with indol, phenol, C5:1 and Fen, negatively correlated with butyric acid. 


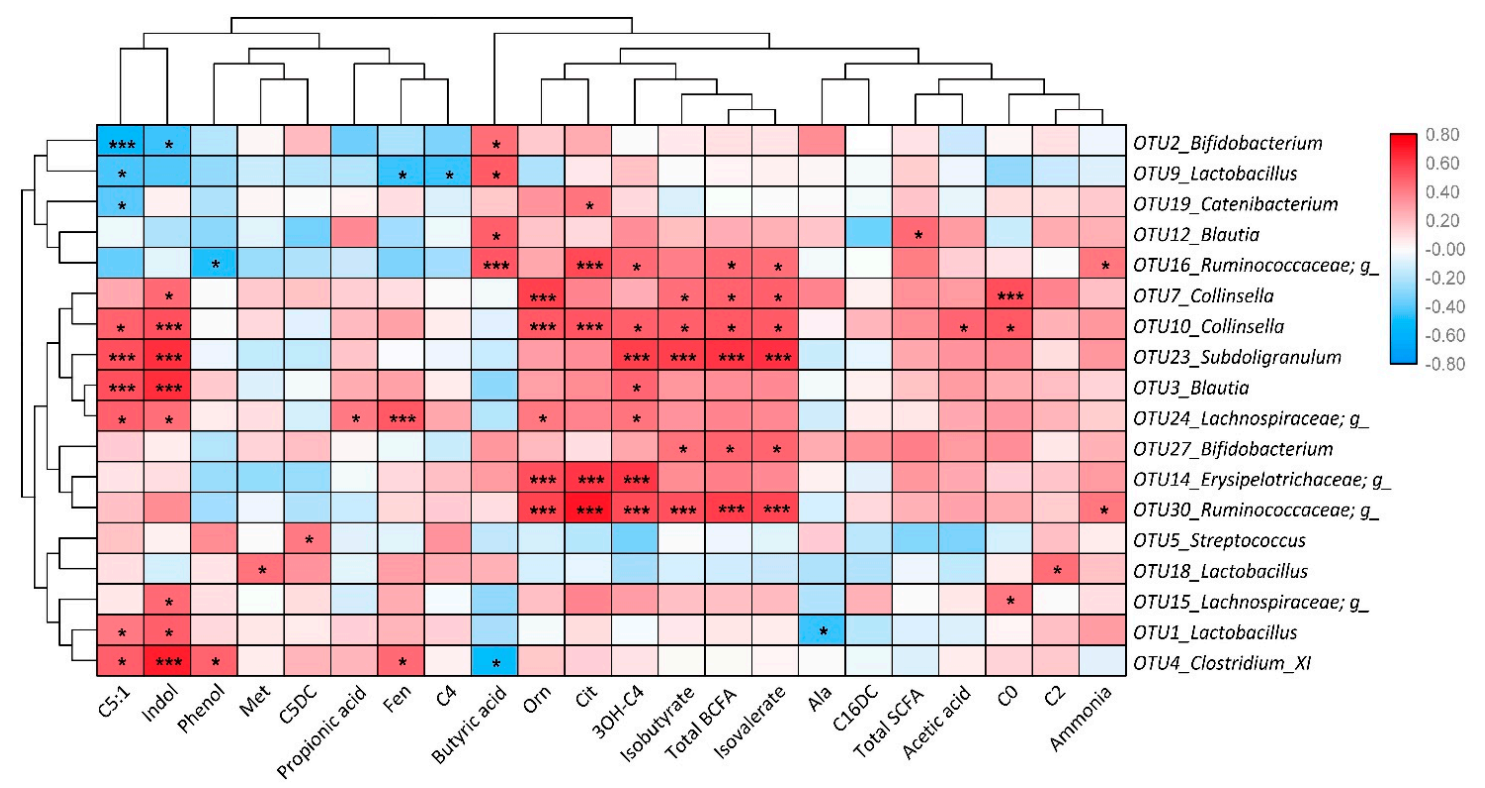

Figure 4. Spearman correlations between OTU abundance and fermentation and metabolism profile. Red represents a positive correlation, while blue represents a negative correlation. "g_" means unclassified genus. ${ }^{*} p<0.05,{ }^{* * *} p<0.01$.

\section{Discussion}

Many studies have investigated the effects of multiple types of prebiotics in cats, such as fructooligosaccharides (FOS) and galactooligosaccharides, using dosage ranging from $0.5 \%$ to $6 \%$ [24-31]. However, the present study showed a different effect, depending on the dose of xylooligosaccharides supplementation. Both XOS treatments influenced the fecal microbiota populations, but the effect of the higher dose was mainly associated with promoting intestinal fermentation, whereas the lower dose was mainly associated with enhancing carbohydrate metabolism.

\subsection{Both XOS Treatments Influenced Intestinal Metabolism and Microbiome}

In this study, both XOS supplemented groups showed a reduction in blood concentrations of $3 \mathrm{OH}-\mathrm{C} 4$ and $\mathrm{C} 16 \mathrm{DC}$. The metabolite $3 \mathrm{OH}-\mathrm{C} 4$ may play an important role in insulin resistance and type 2 diabetes mellitus, as shown in mice [32]. Findings in humans suggested that C16DC was deficient in fibroblasts from patients lacking functional peroxisomes and normal in fibroblasts from patients with a mitochondrial fatty acid oxidation defect [33]. Despite the lack of previous feline data, the present study shows a potential for metabolic regulation in cats by XOS supplementation.

Both XOS groups displayed a lower abundance of Megasphaera and Bifidobacterium and a higher abundance of genera Blautia, Clostridium XI, and Collinsella. The abundance of Bifidobacterium and Megasphaera are often upregulated in humans or dogs with increased carbohydrate fermentation, whereas the abundance of Clostridium XI are often downregulated in these cases [29,33,34]. Findings in this study showed an opposite shift of these bacteria compared to studies in humans or dogs. However, the role of a single bacterium might differ among the gastrointestinal microenvironments from various hosts. For instance, Bifidobacterium has been suggested not to be so important for cat health as for dogs or humans [35]. Blautia belongs to the family of Lachnospiraceae, and herbivores commonly harbor a higher abundance of Lachnospiraceae than carnivores [36]. On the other hand, studies demonstrated that Collinsella could modify host bile acids, alter intestinal cholesterol absorption, decrease glycogenesis, and increase triglyceride synthesis [37,38]. An increased Collinsella abundance in this study may demonstrate a likely association between XOS supplementation and lipid metabolism. Notably, positive correlations between Collinsella and several metabolites and fermentation products were also shown in the present study, indicating a potential for metabolic regulation by microbiota 
induced by prebiotic supplementation. These results suggest that XOS supplementation can influence the environment and ecosystem in the feline gut, but future studies are warranted to unravel underlying mechanisms and specify potential implications.

\subsection{High XOS Supplementation could Promote Intestinal Fermentation}

HXOS group had higher species richness and a more distinct microbiome profile than LXOS or the control group. Species richness promotes stability of microbiota upon increased dietary fiber intake [39], which is a positive effect of $0.4 \%$ XOS supplementation. Additionally, HXOS group increased the abundance of unclassified Ruminococcaceae genus, Erysipelotrichaceae, and Subdoligranulum. Increasing abundance of Erysipelotrichaceae was associated with diet-induced obesity by a high-fat diet in mice $[40,41]$, yet its fluctuation by prebiotics was previously not yet investigated. The cats in the present study were lean both at the start and end of the study. Subdoligranulum belongs to the family Ruminococcaceae, which are common bacteria in the gut that help the host degrade complex carbohydrate-like prebiotics [42]. Moreover, Subdoligranulum and Ruminococcaceae showed positive correlations with SCFAs and BCFAs, emphasizing the interaction between XOS, microbiota shifts, and intestinal fermentation. These results suggested that a $0.4 \%$ XOS supplementation could alter bacterial populations that possibly promote intestinal fermentation.

\subsection{Low XOS Supplementation could Enhance Carbohydrate Metabolism}

In addition to the reduction of $3 \mathrm{OH}-\mathrm{C} 4$ and $\mathrm{C} 16 \mathrm{DC}$, the LXOS group also decreased C5DC concentration in blood, suggesting that dietary $0.04 \%$ XOS supplementation could affect host metabolism in cats [43]. Alterations in these parameters implied that the lower dose XOS might influence carbohydrate metabolism [32,33]. However, very few studies revealed a relationship between C5DC and any metabolic factor in cats and humans [44]. LXOS group also increased the abundance of Streptococcus and Lactobacillus, decreased the abundance of Catenibacterium, suggesting a beneficial effect on the regulation of intestinal ecosystem and metabolism, as Lactobacillus has been highlighted as a beneficial gut bacterium $[45,46]$, and Streptococcus plays a prominent role in the primary carbohydrate metabolism [47]. Catenibacterium is a Gram-positive bacterium from the family Erysipelotrichidae, which has been associated with metabolic disorders in other hosts [48]. Additionally, correlations were observed between C5:1 concentration and Lactobacillus and Catenibacterium as well as between C5DC concentration and Streptococcus, indicating the potential connection between XOS, microbiota, and metabolism. These results suggested that a $0.04 \%$ XOS supplementation could alter microbiota associated with carbohydrate metabolism and modulate host metabolism.

\subsection{Could a Low Dose be Better?}

XOS had no negative effect on the fecal scores at both doses in this study. This is in contrast with some other types of prebiotics that sometimes show an increased fecal moisture content or soft feces [24-26]. The softer feces could indicate a too intensive fermentation [30], but the present study showed that even the high dose $(0.4 \%)$ is safe for use in practice. Often, a decreased fecal $\mathrm{pH}$ is expected with prebiotic supplementation due to the increased production of SCFAs and lactic acid by carbohydrate fermentation [24]. Interestingly, an increased $\mathrm{pH}$ was observed in the LXOS group. However, there was no significant difference when supplementing XOS at $0.4 \%$, suggesting that the expected $\mathrm{pH}$ reduction may only occur at higher doses.

For the first time, this feline study observed a dose-based variation of prebiotic effects and also showed significant differences with a dose as low as $0.04 \%$. Increased concentrations (6-9\%) of fermentable fibers have led to diarrhea $[49,50]$, while reduced concentrations $(<1 \%)$ of fermentable fibers demonstrated positive effects on modulation of microbial community and body metabolism [51,52]. Notably, supplementation of $0.4 \%$ XOS was defined as a 'high dose' in this research, whereas this dosage is substantially lower than in other prebiotic studies. In human clinical trials, health benefits of XOS have typically been observed at $1-4 \mathrm{~g} / \mathrm{d}$, a lower dose than required for prebiotics 
such as fructooligosaccharides and inulin [53]. These results have raised a surprising question and hypothesis_could a low dose be better? And what is the minimal dose required to have prebiotic effects without having side-effects in terms of bloating, soft feces, or other effects? This is warranted to be explored in the future.

\section{Materials and Methods}

\subsection{Animals and Diets}

This study was approved by the Ethical Committee of the Faculty of Veterinary Medicine, Ghent University, Belgium (EC 2015/72), and was in accordance with institutional and national guidelines for the care and use of laboratory animals. Twenty-four adult domestic shorthair cats (8 males and 16 females, all neutered) with a mean age of 8.3 (standard deviation: \pm 2.7 ) years, body weight (BW) of 3.5 (standard deviation: \pm 0.8$) \mathrm{kg}$, and body condition score (BCS, using a 9-point scale) of 4.8 (standard deviation: \pm 0.8 ) were involved in this study. After an adaptation period of three weeks, the cats were equally divided into three groups (two treatment diets and one control diet) considering an equal division of age, gender, and body weight (eight cats per group).

In order to maximize the effects of XOS, a $36 \%$ wheat-free extruded non-commercial diet without additional soluble fiber sources, based on dehydrated poultry meat and corn, was manufactured (ATM Petfood Co., Longué-Jumelles, France). Cellulose and/or XOS 35P (Longlive Biotechnology Ltd., Dezhou, China) were included in order to obtain three different isocaloric and isofibrous diets: LXOS, with a low concentration of XOS $(0.04 \%$ XOS and $0.96 \%$ cellulose); HXOS, high in XOS $(0.40 \%$ XOS and $0.60 \%$ cellulose) and control, XOS free diet with $1 \%$ of cellulose. The dietary ingredients and nutrient composition are depicted in Table 3.

Table 3. Ingredients and macronutrient composition of control and experimental diets.

\begin{tabular}{cccc}
\hline Ingredient (\%) & Control & LXOS & HXOS \\
\hline Corn & 39.11 & 39.11 & 39.11 \\
Dehydrated poultry meat & 19.80 & 19.80 & 19.80 \\
Corn gluten meal & 9.90 & 9.90 & 9.90 \\
Poultry Fat & 6.93 & 6.93 & 6.93 \\
Rice & 4.95 & 4.95 & 4.95 \\
Soy & 4.95 & 4.95 & 4.95 \\
Hydrolyzed dehydrated poultry meat & 4.95 & 4.95 & 4.95 \\
Premix & 2.48 & 2.48 & 2.48 \\
Fishmeal & 2.23 & 2.23 & 2.23 \\
Liquid aroma & 1.98 & 1.98 & 1.98 \\
Yeast & 1.24 & 1.24 & 1.24 \\
Aroma powder & 0.50 & 0.50 & 0.50 \\
Cellulose & 1.00 & 0.96 & 0.60 \\
XOS & 0.00 & 0.040 & 0.40 \\
\hline Analysis on DM basis (\%) & Control & LXOS & HXOS \\
\hline Crude protein & 33.90 & 32.89 & 36.22 \\
Crude fat & 11.49 & 11.56 & 12.14 \\
Crude ash & 6.80 & 6.26 & 5.90 \\
Crude fiber & 1.57 & 1.42 & 1.47 \\
Total dietary fiber & 10.50 & 10.93 & 11.51 \\
Nitrogen free extract & 46.24 & 47.87 & 44.27 \\
\hline
\end{tabular}

All cats were initially adapted to the control diet (1\% cellulose) for three weeks and were fed individually once a day (at 8:00 AM). The amounts of food were calculated according to their individual maintenance energy requirement (MER) by using the MER for adult spayed cats $(313.6 \mathrm{~kJ} / \mathrm{kg} 0.67)$ as a starting point and adapted until a stable body weight was reached according to weekly weighing 
and performing BCS of the cats [54]. Freshwater was continuously provided by automatic drinking fountains and bowls; both were refreshed daily. After the adaptation period, the cats were equally distributed in three treatment groups $(n=8)$ and fed once a day their assigned diet according to their adjusted MER during a period of four weeks. The trial was carried out in two periods (12 cats each period), considering an equal distribution of the different treatment groups over the two periods.

\subsection{Sample Collection}

In the last week of the test period, samples were collected. Before feeding, $8 \mathrm{~mL}$ of blood was aseptically drawn from the jugular vein. Immediately after collection, blood samples were transferred to Vacutainer ${ }^{\circledR}$ tubes (BD Inc., Franklin Lakes, NJ, USA) containing a serum clot activator and lithium heparin. Serum and plasma were separated by centrifugation $(3722 \times g$ for $5 \mathrm{~min})$ and frozen $\left(-20^{\circ} \mathrm{C}\right)$. After blood collection, cats were individually housed with an empty litter box until defecation. To train the cats to use an empty litter box, litter boxes with gradually decreased amounts of litter were provided during the adaptation period. Fresh fecal samples were collected within $15 \mathrm{~min}$ after defecation. The samples were scored for fecal consistency $(1=$ watery liquid that can be poured; $2=$ soft, unformed stool that assumes the shape of the recipient; $3=$ soft, formed, moist stool; $4=$ hard, formed, dry stool; $5=$ hard, dry), fecal $\mathrm{pH}$ was measured with a portable $\mathrm{pH}$ meter (Hanna Instruments Ltd., Temse, Belgium) and samples were immediately frozen thereafter and stored at $-20^{\circ} \mathrm{C}$ until further analysis for fermentation markers and microbiome.

\subsection{Analytical Methods}

Tested diets were subjected to proximate and total dietary fiber analysis (Table 1). Dry matter (DM) was determined by drying to a constant weight at $103^{\circ} \mathrm{C}$, crude protein by calculating from Kjeldahl nitrogen (ISO 5983-1, 2005); crude fat, by the Soxhlet method (ISO 1443, 1973); crude fiber, by acid-alkali digestion (ISO 5498,1981 ) and crude ash by combustion at $550^{\circ} \mathrm{C}$. Nitrogen-free extract was calculated by deducting crude protein, crude fat, crude fiber, and crude ash on a DM basis from 100 . Total dietary fiber was determined by a commercial enzymatic digestion test kit (Sigma-Aldrich Co., St. Louis, MO, USA) and corrected for protein and ash.

Fecal samples were analyzed for SCFAs, branched-chain fatty acids (BCFAs), ammonia, indole $\mathrm{p}$-cresol, phenol, and microbiota. For the analysis of SCFA and BCFA, samples were thawed, and $1 \mathrm{~g}$ of fecal material was transferred into a centrifuge tube together with $5 \mathrm{~mL}$ of water/phosphoric acid/formic acid (550:10:1, v:v:v). Samples were shaken manually before centrifugation (15 $\mathrm{min}$ at 22,000 $\mathrm{g}$ and at $4{ }^{\circ} \mathrm{C}$, Beckman J2-HS, Palo Alto, CA, USA), after which the supernatant was filtered through glass wool prior to GC analysis according to Andersen et al., (2014) [54]. Fecal samples for indole, phenol, and p-cresol analysis $(1 \mathrm{~g})$ were ground, homogenized, and spiked with $100 \mu \mathrm{l}$ of internal standard $(100 \mu \mathrm{g} / \mathrm{mL} 5$-methylindole). Extraction was performed by adding $15 \mathrm{~mL}$ of hexane followed by $10 \mathrm{~min}$ of ultrasonic vibration, $10 \mathrm{~min}$ of rotation (130 tpm), and further ultrasonic vibration for $10 \mathrm{~min}$. After clarification at $4{ }^{\circ} \mathrm{C}$, the supernatant was decanted, and the fecal matter was extracted a second time with $15 \mathrm{~mL}$ of hexane and subsequent ultrasonic vibration and rotation. Supernatants were pooled and centrifuged at $14,000 \mathrm{~g}$ for $10 \mathrm{~min}$ at $4{ }^{\circ} \mathrm{C}$, and $200 \mu \mathrm{L}$ of this combined supernatant was transferred to a GC vial for analysis. All chromatographic analyses were performed with a trace gas chromatograph coupled to a Polaris Q quadrupole ion trap mass spectrometer and a Finnigan MAT A200S autosampler (Thermo-Finnigan, Austin, TX, USA) according to Rochus et al. (2014) [20]. Ammonia nitrogen was analyzed by steam distillation according to standard methods (4500-NH3 B; APHA, 1992).

Blood was analyzed for amino acid profiles and acylcarnitines profiles. A single $3.2 \mathrm{~mm}$ dried blood spot (equivalent to $3.1 \mathrm{~mL}$ of blood) was placed in a polypropylene microtiter plate (Corning). Methanolic internal standard solution $(100 \mathrm{~mL})$ was added either manually or with a Labsystems Multi-drop Dispenser. The concentrations of the deuterium-labeled internal standards per litter of methanol were described by Zytkovicz et al. (2001) [55]. The microtiter plate was gently shaken during the 20-min extraction of the amino acid and acylcarnitine markers. The methanol extract was 
then manually transferred to a second polypropylene microtiter plate and dried by a hot air blower. Butanol- $\mathrm{HCl}(70 \mathrm{~mL})$ was manually placed in each sample well, and the microtiter plate covered with a thin Teflon sheet under a heavy weight and placed in $65^{\circ} \mathrm{C}$ forced air oven for $15 \mathrm{~min}$. After the plate was removed from the oven, the hot air blower removed the butanol- $\mathrm{HCl}$. The butanol-derivatized samples were reconstituted with $100 \mathrm{~mL}$ of acetonitrile and water (80:20 v:v), and each plate was covered with aluminum foil. The samples were then ready for MS/MS analysis, as described in [55].

For microbiota determination, fecal samples were homogenized, and total bacterial DNA was extracted according to Becker et al. (2014) [56]. Twenty-five grams (wet weight) of each thawed fecal sample was placed separately in sterile stomacher bags and homogenized in $225 \mathrm{~mL}$ peptone-buffered saline (PBS) $(0.1 \% w / v$ bacteriological peptone, $0.85 \% w / v \mathrm{NaCl})$. The sludgy homogenate was filtered on a Büchner funnel to discard large particles such as hair and bones and subsequently divided into $1.5 \mathrm{~mL}$ aliquots, which were stored at $-80^{\circ} \mathrm{C}$. The protocol of Vanhoutte et al. (2004) was used to extract total bacterial DNA from the fecal samples [57]. DNA size and integrity were assessed on $1 \%$ agarose electrophoresis gels stained with ethidium bromide. DNA concentration and purity were determined by spectrophotometric measurement at $234 \mathrm{~nm}, 260 \mathrm{~nm}$, and $280 \mathrm{~nm}$. Subsequently, DNA samples were sent to the company LGC Genomics (Teddington, UK) for library preparation, sequencing (Illumina Miseq), and bioinformatic analysis. 16S rRNA gene sequences were obtained from the SILVA database (Max Planck Institute for Marine Microbiology and Jacobs University, Bremen, Germany), and analysis of bioinformation was processed as described by Camarinha-Silva et al. (2014) [58].

\subsection{Statistical Analysis}

The statistical analysis was conducted in $\mathrm{R}$ version 4.0.2. Significance was set at $\alpha \leq 0.05$, and a $p$-value $\leq 0.1$ was considered a trend. Data are reported as median and range. To assess the effect of diet, a linear model was used with diet as an independent variable and the various fecal and blood parameters as dependent variables. The significance of the dependent variable was assessed with a likelihood ratio test. When significant, a Tukey Honest Significant Differences test was conducted as a post-hoc test.

For the microbiome data, Shannon and (inverse) Simpson indexes, Pielou's evenness, and species richness were analyzed for microbial diversity. Significant differences in bacterial abundance were evaluated using the analysis of similarities (ANOSIM). All microbiome data evaluations were performed by the online platform MicrobiomeAnalyst (McGill University, Montreal, QC, Canada). Additionally, using SPSS v24.0 software, spearman correlations coefficients were calculated between the fecal microbiome and fecal fermentation, plasma amino acids, and acylcarnitine profiles. The $p$-values were corrected with the false discovery rate method.

\section{Conclusions}

This study investigated the effect of two doses of XOS supplementation in healthy adult cats. The major finding was that both two levels of XOS affected the fecal microbiome in cats. Surprisingly, this study demonstrated a different effect, depending on the supplemented dose. The impact of the lower-dose XOS was mainly associated with carbohydrate metabolism, while the higher dose was mainly associated with intestinal fermentation. These findings indicated that supplementation XOS could benefit the gut health of cats by influencing microbiota and intestinal metabolism, while its different effects depend on the dosage. Future research is needed to further explore the association between the effects and doses of the supplementation of XOS as well as other prebiotics. Moreover, significant effects were observed even with a very low dose of XOS, pointing towards more research on the low dosage of prebiotics in the future.

Supplementary Materials: The following are available online, Table S1: Supplementary data.

Author Contributions: Conceptualization, M.H., H.B. and M.R.; methodology, B.J.G.B., L.V., and T.V.d.W.; formal analysis, Y.L. and S.D.; investigation, Y.L. and S.D.; data curation, Y.L.; writing-original draft preparation, Y.L.; 
writing—review and editing, M.H., L.V., and T.V.d.W.; supervision, M.H. All authors have read and agreed to the published version of the manuscript.

Funding: This work was supported by the Longlive Biotechnology Company (Qingdao, China).

Acknowledgments: The authors would like to thank Herman De Rycke, Amy De Luycker, and Liesbeth Timmerman for technical assistance. The Lab of Chemical Analysis research group is part of the Ghent University expertise centre MSsmall.

Conflicts of Interest: The authors declare no conflict of interest.

\section{References}

1. Esgalhado, M.; Kemp, J.A.; Damasceno, N.R.; Fouque, D.; Mafra, D. Short-chain fatty acids: A link between prebiotics and microbiota in chronic kidney disease. Future Microbiol. 2017, 12, 1413-1425. [CrossRef] [PubMed]

2. Dewulf, E.M.; Cani, P.D.; Claus, S.P.; Fuentes, S.; Puylaert, P.G.; Neyrinck, A.M.; Bindels, L.B.; de Vos, W.M.; Gibson, G.R.; Thissen, J.P.; et al. Insight into the prebiotic concept: Lessons from an exploratory, double blind intervention study with inulin-type fructans in obese women. Gut 2013, 62, 1112-1121. [CrossRef] [PubMed]

3. Cani, P.D.; Lecourt, E.; Dewulf, E.M.; Sohet, F.M.; Pachikian, B.D.; Naslain, D.; De Backer, F.; Neyrinck, A.M.; Delzenne, N.M. Gut microbiota fermentation of prebiotics increases satietogenic and incretin gut peptide production with consequences for appetite sensation and glucose response after a meal. Am. J. Clin. Nutr. 2009, 90, 1236-1243. [CrossRef] [PubMed]

4. Ghouri, Y.; Richards, M.; Rahimi, E.; Krill, J.; Jelinek, K.A.; Dupont, A.W. Systematic review of randomized controlled trials of probiotics, prebiotics, and synbiotics in inflammatory bowel disease. Clin. Exp. Gastroenterol. 2014, 7, 473-487.

5. Respondek, F.; Swanson, K.S.; Belsito, K.R.; Vester, B.M.; Wagner, A.; Istasse, L.; Diez, M. Short-chain fructooligosaccharides influence insulin sensitivity and gene expression of fat tissue in obese dogs. J. Nutr. 2008, 138, 1712-1718. [CrossRef] [PubMed]

6. Respondek, F.; Myers, K.; Smith, T.L.; Wagner, A.; Geor, R.J. Dietary supplementation with short-chain fructo-oligosaccharides improves insulin sensitivity in obese horses. J. Anim. Sci. 2011, 89, 77-83. [CrossRef] [PubMed]

7. Che, T.M.; Johnson, R.W.; Kelley, K.W.; Van Alstine, W.G.; Dawson, K.A.; Moran, C.A.; Pettigrew, J.E. Mannan oligosaccharide modulates gene expression profile in pigs experimentally infected with porcine reproductive and respiratory syndrome virus. J. Anim. Sci. 2011, 89, 3016-3029. [CrossRef] [PubMed]

8. Cho, J.H.; Kim, I.H. Effects of lactulose supplementation on performance, blood profiles, excreta microbial shedding of Lactobacillus and Escherichia coli, relative organ weight and excreta noxious gas contents in broilers. J. Anim. Physiol. Anim. Nutr. 2014, 98, 424-430. [CrossRef]

9. Vázquez, M.J.; Alonso, J.L.; Domínguez, H.; Parajó, J.C. Xylooligosaccharides: Manufacture and applications. Trends Food Sci. Tech. 2000, 11, 387. [CrossRef]

10. Jain, I.; Kumar, V.; Satyanarayana, T. Xylooligosaccharides: An economical prebiotic from agroresidues and their health benefits. Indian J. Exp. Biol. 2015, 53, 131-142.

11. Aachary, A.A.; Prapulla, S.G. Xylooligosaccharides (xos) as an emerging prebiotic: Microbial synthesis, utilization, structural characterization, bioactive properties, and applications. Compr. Rev. Food Sci. Food Saf. 2011, 10, 2-16. [CrossRef]

12. Broekaert, W.F.; Courtin, C.M.; Verbeke, K.; Van de Wiele, T.; Verstraete, W.; Delcour, J.A. Prebiotic and other health-related effects of cereal-derived arabinoxylans, arabinoxylan-oligosaccharides, and xylooligosaccharides. Crit. Rev. Food Sci. Nutr. 2011, 51, 178-194. [CrossRef] [PubMed]

13. Gobinath, D.; Madhu, A.N.; Prashant, G.; Srinivasan, K.; Prapulla, S.G. Beneficial effect of xylo-oligosaccharides and fructo-oligosaccharides in streptozotocin-induced diabetic rats. Brit. J. Nutr. 2010, 104, 40-47. [CrossRef] [PubMed]

14. Imaizumi, K.; Nakatsu, Y.; Sato, M.; Sedarnawati, Y.; Sugano, M. Effects of xylooligosaccharides on blood glucose, serum and liver lipids and cecum short-chain fatty acids in diabetic rats. Agri. Biol. Chem. 1991, 55, 199-205.

15. Sheu, H.H.; Lee, I.T.; Chen, W.; Chan, Y.C. Effects of xylooligosaccharides in type 2 diabetes mellitus. J. Nutr. Sci. Vitaminol. 2008, 54, 396-401. [CrossRef] [PubMed] 
16. Na, M.; Kim, W. Effects of xylooligosaccharide intake on fecal Bifidobacteria, lactic acid and lipid metabolism in Korean young women. Korean J. Nutr. 2007, 40, 154-161.

17. Bouhnik, Y.; Vahedi, K.; Achour, L.; Attar, A.; Salfati, J.; Pochart, P.; Marteau, P.; Flourié, B.; Bornet, F.; Rambaud, J.C. Short-chain fructo-oligosaccharide administration dose-dependently increases fecal bifidobacteria in healthy humans. J. Nutr. 1999, 129, 113-116. [CrossRef] [PubMed]

18. Ito, M.; Deguchi, Y.; Miyamori, A.; Matsumoto, K.; Kikuchi, H.; Matsumoto, K.; Kobayashi, Y.; Yajima, T.; Kan, T. Effects of Administration of Galactooligosaccharides on the Human Faecal Microflora, Stool Weight and Abdominal Sensation. Microb. Ecol. Health Dis. 1990, 3, 285-292.

19. Lin, X.; Ning, J.; Xu, G. Application of Xylo-oligosaccharide in modifying human intestinal function. Afr. J. Microbiol. Res. 2012, 6, 2116-2119.

20. Rochus, K.; Janssens, G.P.; Hesta, M. Dietary fibre and the importance of the gut microbiota in feline nutrition: A review. Nutr. Res. Rev. 2014, 27, 295-307. [CrossRef]

21. Brosey, B.P.; Hill, R.C.; Scott, K.C. Gastrointestinal volatile fatty acid concentrations and pH in cats. Am. J. Vet. Res. 2000, 61, 359-361. [CrossRef] [PubMed]

22. Zoran, D.L. The carnivore connection to nutrition in cats. J. Am. Vet. Med. Assoc. 2002, 221, $1559-1567$. [CrossRef] [PubMed]

23. Verbrugghe, A.; Hesta, M.; Daminet, S.; Janssens, G. Nutritional modulation of insulin resistance in the true carnivorous cat: A review. Crit. Rev. Food Sci. Nutr. 2012, 52, 172-182. [CrossRef] [PubMed]

24. Hesta, M.; Janssens, G.P.J.; Debraekeleer, J.; Wilde, R.D. The effect of oligofructose and inulin on faecal characteristics and nutrient digestibility in healthy cats. J. Anim. Physiol. Anim. Nutr. 2001, 85, 7. [CrossRef] [PubMed]

25. Hesta, M.; Hoornaert, E.; Verlinden, A.; Janssens, G.P.J. The effect of oligofructose on urea metabolism and faecal odour components in cats. J. Anim. Physiol. Anim. Nutr. 2005, 89, 7. [CrossRef] [PubMed]

26. Verbrugghe, A.; Hesta, M.; Gommeren, K.; Daminet, S.; Wuyts, B.; Buyse, J.; Janssens, G.P.J. Oligofructose and inulin modulate glucose and amino acid metabolism through propionate production in normal-weight and obese cats. Brit. J. Nutr. 2009, 102, 694. [CrossRef] [PubMed]

27. Verbrugghe, A.; Hesta, M.; Van Weyenberg, S.; Papadopoulos, G.A.; Gommeren, K.; Daminet, S.; Bosmans, T.; Polis, I.; Buyse, J.; Janssens, G.P.J. The glucose and insulin response to isoenergetic reduction of dietary energy sources in a true carnivore: The domestic cat (felis catus). Br. J. Nutr. 2010, 104, 214-221. [CrossRef]

28. Kanakupt, K.; Vester Boler, B.M.; Dunsford, B.R.; Fahey, G.C., Jr. Effects of short-chain fructooligosaccharides and galactooligosaccharides, individually and in combination, on nutrient digestibility, fecal fermentative metabolite concentrations, and large bowel microbial ecology of healthy adult cats. J. Anim. Sci. 2011, 89, 1376-1384. [CrossRef]

29. Barry, K.A.; Wojcicki, B.J.; Middelbos, I.S.; Vester, B.M.; Swanson, K.S.; Fahey, G.C. Dietary cellulose, fructooligosaccharides, and pectin modify fecal protein catabolites and microbial populations in adult cats. J. Anim. Sci. 2010, 88, 2978-2987. [CrossRef]

30. Barry, K.A.; Middelbos, I.S.; Vester Boler, B.M.; Dowd, S.E.; Suchodolski, J.S.; Henrissat, B.; Coutinho, P.M.; White, B.A.; Fahey, G.C., Jr.; Swanson, K.S. Effects of dietary fiber on the feline gastrointestinal metagenome. J. Proteome Res. 2012, 11, 5924-5933. [CrossRef]

31. Barry, K.A.; Hernot, D.C.; Van Loo, J.; Fahey, G.C.; de Godoy, M.R.C. Fructan supplementation of senior cats affects stool metabolite concentrations and fecal microbiota concentrations, but not nitrogen partitioning in excreta. J. Anim. Sci. 2014, 92, 4964-4971. [CrossRef] [PubMed]

32. An, J.; Muoio, D.M.; Shiota, M.; Fujimoto, Y.; Cline, G.W.; Shulman, G.I.; Koves, T.R.; Stevens, R.; Millington, D.; Newgard, C.B. Hepatic expression of malonyl-CoA decarboxylase reverses muscle, liver and whole-animal insulin resistance. Nat. Med. 2004, 10, 268. [CrossRef] [PubMed]

33. Ferdinandusse, S.; Denis, S.; Mooijer, P.A.; Zhang, Z.; Reddy, J.K.; Spector, A.A.; Wanders, R.J. Identification of the peroxisomal $\beta$-oxidation enzymes involved in the biosynthesis of docosahexaenoic acid. J. Lipid Res. 2001, 42, 1987-1995. [PubMed]

34. O'Keefe, S.J. Tube feeding, the microbiota, and Clostridium difficile infection. World J. Gastroenterol. 2010, 16, 139. [CrossRef]

35. Masuoka, H.; Shimada, K.; Kiyosue-Yasuda, T.; Kiyosue, M.; Oishi, Y.; Kimura, S.; Ohashi, Y.; Fujisawa, T.; Hotta, K.; Yamada, A.; et al. Transition of the intestinal microbiota of cats with age. PLoS ONE 2017, 12, e0181739. [CrossRef] 
36. Furet, J.P.; Firmesse, O.; Gourmelon, M.; Bridonneau, C.; Tap, J.; Mondot, S.; Dore, J.; Corthier, G. Comparative assessment of human and farm animal faecal microbiota using real-time quantitative PCR. FEMS Microbiol. Ecol. 2009, 68, 351-362. [CrossRef]

37. Lahti, L.; Salonen, A.; Kekkonen, R.A.; Salojärvi, J.; Jalanka-Tuovinen, J.; Palva, A.; Orešič, M.; De Vos, W.M. Associations between the human intestinal microbiota, Lactobacillus rhamnosus GG and serum lipids indicated by integrated analysis of high-throughput profiling data. Peer J. 2013, 1, e32. [CrossRef]

38. Bag, S.; Ghosh, T.S.; Das, B. Complete genome sequence of Collinsella aerofaciens Isolated from the gut of a healthy Indian subject. Genome Announc. 2017, 5, e01361-17. [CrossRef]

39. Tap, J.; Furet, J.P.; Bensaada, M.; Philippe, C.; Roth, H.; Rabot, S.; Lakhdari, O.; Lombard, V.; Henrissat, B.; Corthier, G.; et al. Gut microbiota richness promotes its stability upon increased dietary fibre intake in healthy adults. Environ. Microbiol. 2015, 17, 4954-4964. [CrossRef]

40. Turnbaugh, P.J.; Backhed, F.; Fulton, L.; Gordon, J.I. Diet-induced obesity is linked to marked but reversible alterations in the mouse distal gut microbiome. Cell Host Microbe 2008, 3, 213-223. [CrossRef]

41. Daniel, H.; Gholami, A.M.; Berry, D.; Desmarchelier, C.; Hahne, H.; Loh, G.; Mondot, S.; Lepage, P.; Rothballer, M.; Walker, A.; et al. High-fat diet alters gut microbiota physiology in mice. ISME J. 2014, 8, 295. [CrossRef] [PubMed]

42. Flint, H.J.; Scott, K.P.; Duncan, S.H.; Louis, P.; Forano, E. Microbial degradation of complex carbohydrates in the gut. Gut Microbes 2012, 3, 289-306. [CrossRef]

43. Yu, Z.R.; Ning, Y.; Yu, H.; Tang, N.J. A HPLC-Q-TOF-MS-based urinary metabolomic approach to identification of potential biomarkers of metabolic syndrome. J. Huazhong Univ. Sci. Technol. Med. Sci. 2014, 34, $276-283$. [CrossRef] [PubMed]

44. Barclay, K.D.; McKersie, B.D. Peroxidation reactions in plant membranes: Effects of free fatty acids. Lipids 1994, 29, 877-882. [CrossRef] [PubMed]

45. Coxam, V. Current data with inulin-type fructans and calcium, targeting bone health in adults. J. Nutr. 2007, 137, 2527S-2533S. [CrossRef] [PubMed]

46. Seifert, S.; Watzl, B. Inulin and oligofructose: Review of experimental data on immune modulation. J. Nutr. 2007, 137, 2563S-2567S. [CrossRef]

47. Zoetendal, E.G.; Raes, J.; Van Den Bogert, B.; Arumugam, M.; Booijink, C.C.; Troost, F.J.; Bork, P.; Wels, M.; De Vos, W.M.; Kleerebezem, M. The human small intestinal microbiota is driven by rapid uptake and conversion of simple carbohydrates. ISME J. 2012, 6, 1415. [CrossRef]

48. Kaakoush, N.O. Insights into the Role of Erysipelotrichaceae in the Human Host. Front. Cell. Infect. Microbiol. 2015, 5, 84. [CrossRef]

49. Sunvold, G.D.; Fahey, G.C., Jr.; Merchen, N.R.; Reinhart, G.A. In vitro fermentation of selected fibrous substrates by dog and cat fecal inoculum: Influence of diet composition on substrate organic matter disappearance and short-chain fatty acid production. J. Anim. Sci. 1995, 73, 1110-1122. [CrossRef]

50. Bueno, A.R.; Cappel, T.G.; Sunvold, G.D.; Moxley, R.A.; Reinhart, G.A.; Clemens, E.T. Feline colonic microbes and fatty acid transport: Effects of feeding cellulose, beet pulp and pectin/gum arabic fibers. Nutr. Res. 2000, 20, 1319-1328. [CrossRef]

51. Sparkes, A.H.; Papasouliotis, K.; Sunvold, G.; Werrett, G.; Gruffydd-Jones, E.A.; Egan, K.; Gruffydd-Jones, T.J.; Reinhart, G. Effect of dietary supplementation with fructo-oligosaccharides on fecal flora of healthy cats. Am. J. Vet. Res. 1998, 59, 436-440.

52. Groeneveld, E.A.; Kappert, H.J.; der Kuilen Van, J.; Beynen, A.C. Consumption of fructooligosaccharides and nitrogen excretion in cats. Int. J. Vitam. Nutr. Res. 2001, 71, 254-256. [CrossRef] [PubMed]

53. Saville, B.A.; Saville, S. Xylooligosaccharides and Arabinoxylanoligosaccharides and Their Application as Prebiotics. Appl. Food Biotechnol. 2018, 5, 121-130.

54. Andersen, S.J.; Hennebel, T.; Gildemyn, S.; Coma, M.; Desloover, J.; Berton, J.; Tsukamoto, J.; Stevens, C.; Rabaey, K. Electrolytic membrane extraction enables production of fine chemicals from biorefinery sidestreams. Environ. Sci. Technol. 2014, 48, 7135-7142. [CrossRef] [PubMed]

55. Zytkovicz, T.H.; Fitzgerald, E.F.; Marsden, D.; Larson, C.A.; Shih, V.E.; Johnson, D.M.; Strauss, A.W.; Comeau, A.M.; Eaton, R.B.; Grady, G.F. Tandem mass spectrometric analysis for amino, organic, and fatty acid disorders in newborn dried blood spots: A two-year summary from the New England newborn screening program. Clin. Chem. 2001, 47, 1945-1955. [CrossRef] [PubMed] 
56. Becker, A.A.; Hesta, M.; Hollants, J.; Janssens, G.P.; Huys, G. Phylogenetic analysis of faecal microbiota from captive cheetahs reveals underrepresentation of Bacteroidetes and Bifidobacteriaceae. BMC Microbiol. 2014, 14, 43. [CrossRef] [PubMed]

57. Vanhoutte, T.; Huys, G.; De Brandt, E.; Swings, J. Temporal stability analysis of the microbiota in human feces by denaturing gradient gel electrophoresis using universal and group-specific 16S rRNA gene primers. FEMS Microbiol. Ecol. 2004, 48, 437-446. [CrossRef]

58. Camarinha-Silva, A.; Jáuregui, R.; Chaves-Moreno, D.; Oxley, A.P.; Schaumburg, F.; Becker, K.; Wos-Oxley, M.L.; Pieper, D.H. Comparing the anterior nare bacterial community of two discrete human populations using I llumina amplicon sequencing. Environ. Microbiol. 2014, 16, 2939-2952. [CrossRef] [PubMed]

Sample Availability: Small samples (a few milligrams) of the compounds 8, 12, and 13 are available from the authors.

Publisher's Note: MDPI stays neutral with regard to jurisdictional claims in published maps and institutional affiliations.

(C) 2020 by the authors. Licensee MDPI, Basel, Switzerland. This article is an open access article distributed under the terms and conditions of the Creative Commons Attribution (CC BY) license (http://creativecommons.org/licenses/by/4.0/). 